\title{
10-Gb/s SCM Fiber System Using Optical SSB Modulation
}

\author{
R. Hui, Senior Member, IEEE, B. Zhu, R. Huang, C. Allen, Senior Member, IEEE, K. Demarest, Senior Member, IEEE, \\ and D. Richards
}

\begin{abstract}
A 10-Gb/s subcarrier multiplexing long-haul optical system is reported. $4 \times 2.5 \mathrm{~Gb} / \mathrm{s}$ data streams are combined into one wavelength, which occupies a 20-GHz optical bandwidth. Optical single sideband is used to increase bandwidth efficiency and reduce dispersion penalty. The receiver sensitivity is calculated using a simplified receiver model with an optical preamplifier. The measured results agree well with the analytical prediction.
\end{abstract}

Index Terms-Modulation, optical communication, optical modulation, optical signal processing.

I $\mathrm{N}$ ORDER to use the optical bandwidth provided by optical fibers more efficiently, new transmission technologies have been developed rapidly in recent years, such as time division multiplexing (TDM), wavelength division multiplexing (WDM), and their combinations. Apart from noise accumulation, high-speed TDM optical systems suffer from chromatic dispersion, nonlinear crosstalk, and polarization-mode dispersion (PMD). Optical systems with data rates of $10 \mathrm{~Gb} / \mathrm{s}$ and higher require precise dispersion compensation, and careful link engineering. On the other hand, WDM technology uses lower data rates at each wavelength. However, due to the limitations in the wavelength stability of semiconductor lasers and selectivity of optical filters, the minimum channel spacing is $50 \mathrm{GHz}$ in current commercial WDM systems. Narrower channel spacing requires better wavelength stability of laser sources and better wavelength selectivity of optical filters.

Optical subcarrier multiplexing (SCM) is a scheme where multiple signals are multiplexed in the RF domain and transmitted by a single wavelength. A significant advantage of SCM is that microwave devices are more mature than optical devices: the stability of a microwave oscillator and the frequency selectivity of a microwave filter are much better than their optical counterpart. A popular application of SCM technology in fiber optic systems is analog cable television (CATV) distribution [1], [2]. Because of the simple and low-cost implementation, SCM has also been proposed to transmit multichannel digital-optical signals using direct detection [3], [4] for local area optical networks.

The basic configuration of an SCM/WDM optical system is shown in Fig. 1. In this example, $n$-independent high-speed digital signals are mixed by $n$-different microwave carrier frequencies $f_{i}$. These are combined and optically modulated onto an

\footnotetext{
Manuscript received March 12, 2001; revised May 1, 2001. This work was supported by Sprint Communications Company, L. P.

R. Hui, B. Zhu, R. Huang, C. Allen, and K. Demarest are with the Department of Electrical and Computer Science, Information and Telecommunication Technology Center, University of Kansas, Lawrence, KS 66044 USA.

D. Richards is with Sprint, Overland Park, KS 66212 USA.

Publisher Item Identifier S 1041-1135(01)06410-2.
}

optical carrier. $m$-wavelengths are then multiplexed together in an optical WDM configuration. At the receiver, an optical demultiplexer separates the wavelengths for individual optical detectors. Then, RF coherent detection is used at the SCM level to separate the digital signal channels. Channel add/drop is also possible in both wavelength level and SCM level. While this $\mathrm{SCM} / \mathrm{WDM}$ is in fact an ultradense WDM system, sophisticated microwave and RF technology enables the channel spacing to be comparable to the baseband, which is otherwise not feasible by using optical technology. Compared to conventional high-speed TDM systems, SCM is less sensitive to fiber dispersion, because the dispersion penalty is determined by the width of the baseband of each individual signal channel. Compared to conventional WDM systems, on the other hand, it has better optical spectral efficiency because much narrower channel spacing is allowed.

Conventional SCM generally occupies a wide modulation bandwidth, because of its double-sideband spectrum structure, and, therefore is susceptible to chromatic dispersion. In order to reduce dispersion penalty and increase optical bandwidth efficiency, optical SSB modulation is essential for long-haul SCM/WDM optical systems. Fortunately, optical single sideband (SSB) [5] is relatively easy to accomplish in SCM systems, because there are no low frequency components, and Hilbert transformation is thus, much simpler than in conventional TDM systems [6], [7].

In order to investigate the feasibility of long-haul digital SCM transmission at high speed, an experiment was conducted at $10-\mathrm{Gb} / \mathrm{s}$ capacity per wavelength. Four $2.5-\mathrm{Gb} / \mathrm{s}$ digital signals were mixed with four microwave carriers each at 3.6, 8.3, 13, and $18 \mathrm{GHz}$. They were then combined and amplified to drive a dual-electrode $\mathrm{LiNbO}_{3}$ Mach-Zehnder modulator (MZM) with a 20-GHz bandwidth. As shown in Fig. 2, in order to generate an optical single sideband, the composite signal was applied to both of the two balanced electrodes with a $\pi / 2$ phase shift in one of the arms using a $90^{\circ}$ hybrid splitter. A dc bias sets the modulator at the quadrature point to generate optical SSB [8]. Fig. 3 shows the SSB optical spectrum measured by a scanning Fabry-Perot interferometer with 1-GHz resolution bandwidth.

To measure the transmission performance, this optical signal was then launched into a single-mode fiber link with accumulated chromatic dispersion of $-2640 \mathrm{ps} / \mathrm{nm}$, which is equivalent to approximately $160 \mathrm{~km}$ of standard single-mode fiber. Due to the limitation of fiber availability, the experiment was performed in dispersion compensating fibers (DCF) (Lucent DK series), which have large negative dispersion values. No dispersion compensation was used. At the receiver, the optical signal 
Transmitter 1

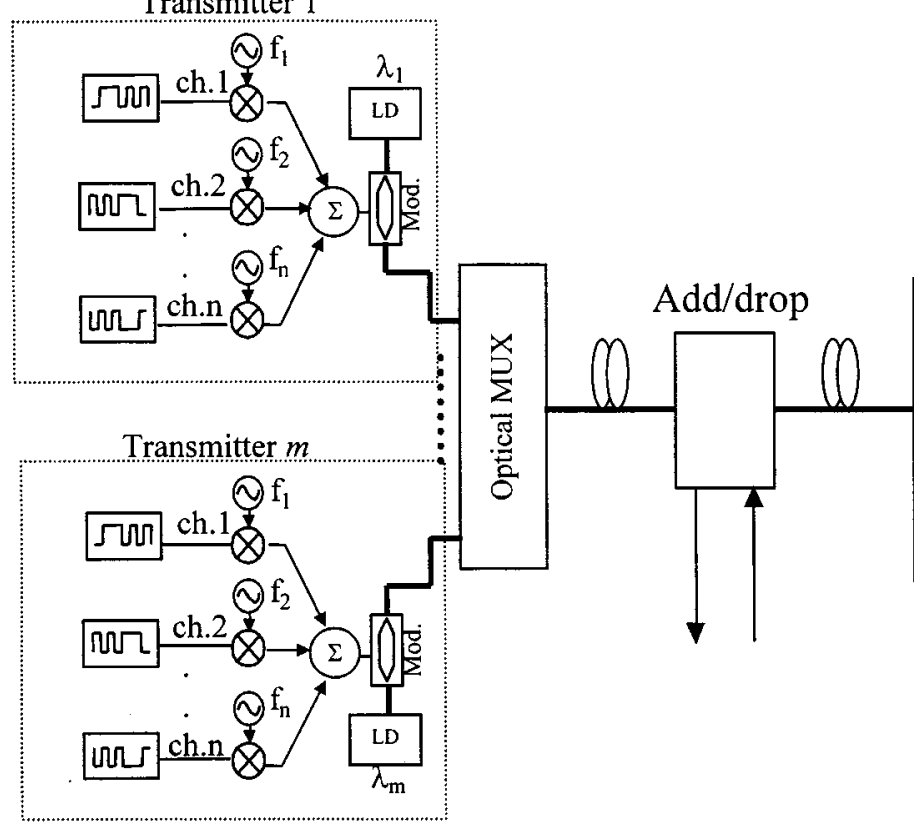

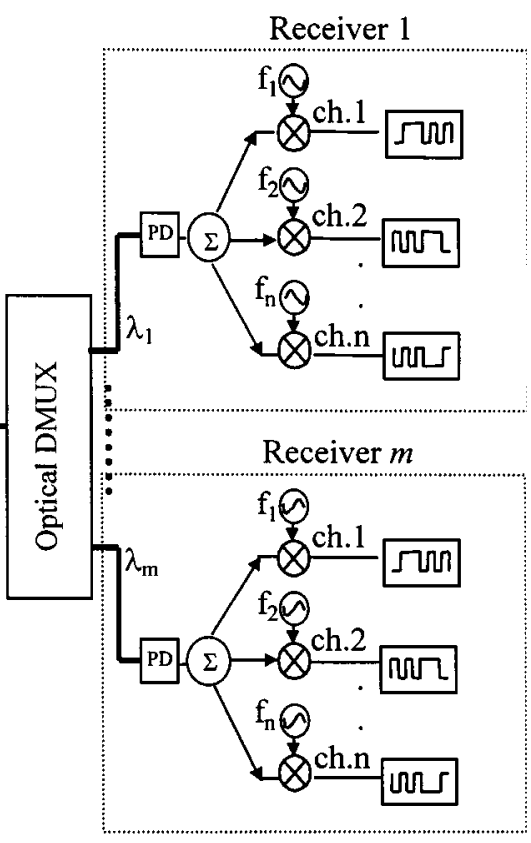

Fig. 1. SCM/WDM system architecture.

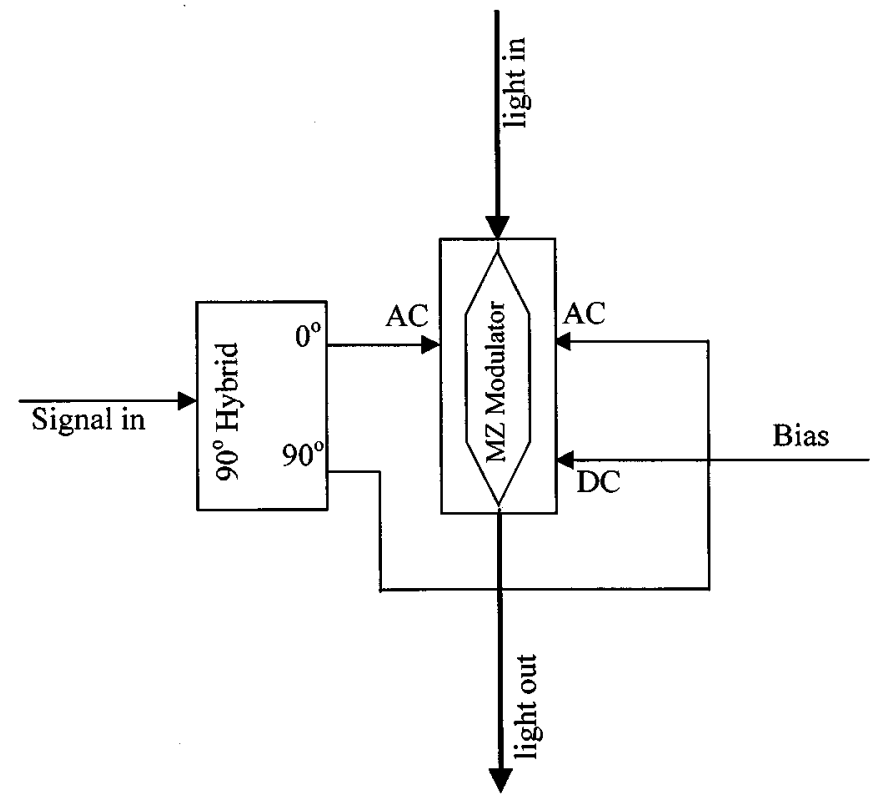

Fig. 2. Optical SSB modulation using dual-electrode MZM.

was preamplified and detected by a wide-band photodetector. The digital signal was then down-converted to each individual baseband by mixing the composite signal with local RF oscillators, and then passing through a $1.75-\mathrm{GHz}$ baseband filter. $\mathrm{Bi}-$ nary phase-shift-keying (PSK) modulation scheme was adopted in the RF domain for better receiver sensitivity. Bit-error rate (BER) was measured for all four channels both back-to-back and over the fiber. The measured BER plotted as a function of received optical power level, which is shown in Fig. 4. The measurement was performed under the condition that all four SCM channels were simultaneously operated. At the BER level of $10^{-10}$, the back-to-back sensitivity ranges from -25 to -27 $\mathrm{dBm}$ for different channels due to the ripples in the microwave

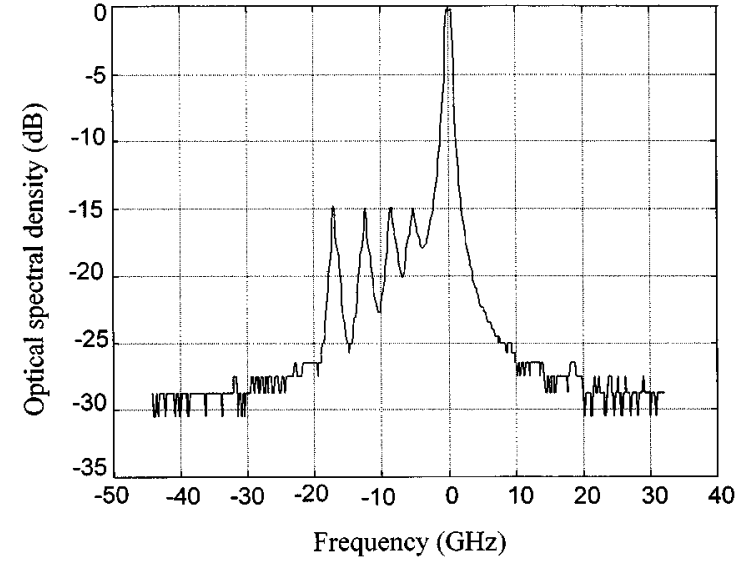

Fig. 3. Measured SSB optical spectrum with four subcarrier channels.

devices and the inaccuracy of the modulation index of each individual SCM channel. After transmission, the sensitivity is degraded by typically $2.5 \mathrm{~dB}$. In our experiment, this degradation was largely attributed to the frequency instability of the local oscillators. Although the minimum allowed channel spacing, largely depends on the quality of the baseband filter, which in our experiment, a $4.7-\mathrm{GHz}$ spacing was necessary. Further reduction in channel spacing resulted in a sharp degradation of BER. Further improvement of bandwidth efficiency can be made using microwave SSB modulation.

Owing to the relative low-data rates carried by each individual SCM channel, the SCM system can tolerate more dispersion than a TDM system of the same capacity. We have made an experimental comparison of the system performance between an OC-192 TDM system and a four-channel OC-48 SCM system. Fig. 5 shows the measured receiver sensitivities versus the accumulated dispersion. At back-to-back, the sensitivity of the SCM system is about $6 \mathrm{~dB}$ worse compared to its TDM counterpart, because of small modulation index in the SCM system. How- 


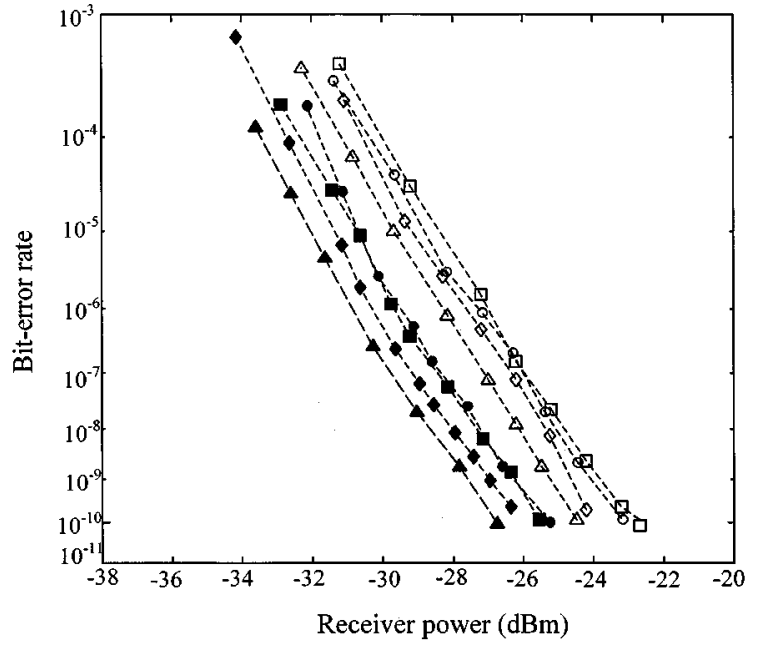

Fig. 4. Measured BER in a system with 4 subcarrier channels before (solid points) and after (open points) a fiber transmission line with a $-2640 \mathrm{ps} / \mathrm{nm}$ total dispersion.

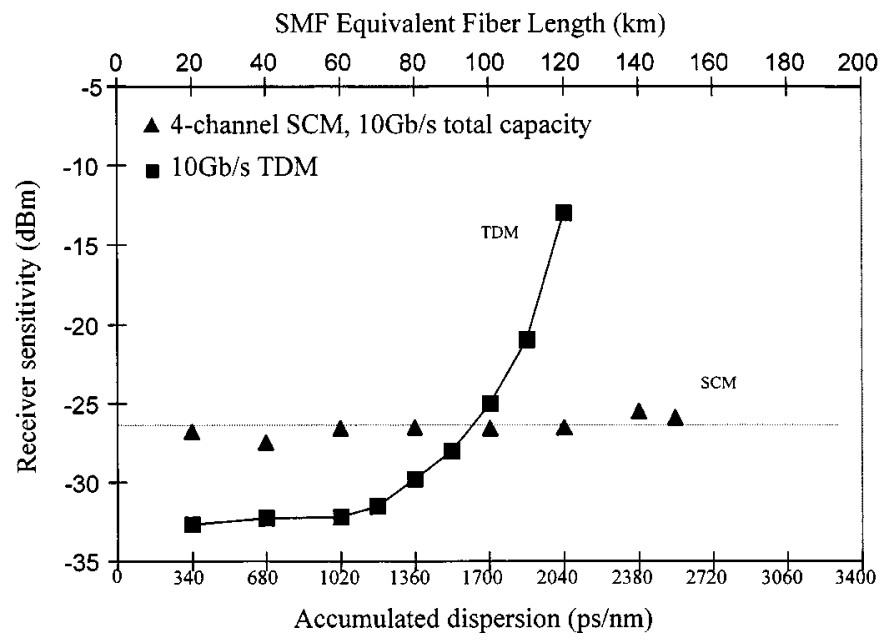

Fig. 5. Receiver sensitivity (at BER of $10^{-9}$ ) comparison between a $10-\mathrm{Gb} / \mathrm{s}$ TDM system and a four-tone SCM system with $10-\mathrm{Gb} / \mathrm{s}$ capacity.

ever, with the accumulated dispersion of higher than $1700 \mathrm{ps} / \mathrm{nm}$ (corresponds to $100 \mathrm{~km}$ of SMF), the performance of the TDM system deteriorates rapidly, while the performance of the SCM system remains unchanged.

For a PSK modulated digital SCM system with an optically preamplified receiver, the system $Q$ value can be derived as

$$
Q=\frac{m_{k} \sqrt{P_{\mathrm{in}}}-(-1) m_{k} \sqrt{P_{\mathrm{in}}}}{2 \sqrt{2 F h v B_{e}}}=\frac{m_{k} \sqrt{P_{\mathrm{in}}}}{\sqrt{2 F h v B_{e}}}
$$

where

$P_{\text {in }} \quad$ optical power entering the photo diode;

$m_{k} \quad$ modulation index with $0<m_{k}<1 / N$ with $N$ the number of subcarrier channels;

$F \quad$ optical amplifier noise figure;

$h \nu$ photon energy;

$B_{e} \quad$ width of the electrical baseband filter.
In deriving this formula, the approximations used were: 1) no waveform distortion; 2) signal-amplified spontaneous emission (ASE) beat noise is the dominant noise, which is valid for receivers with optical preamplifier; 3) optical modulation response of the modulator was linearized, which is valid for small-signal modulation; 4) double-sideband ASE noise, assuming the receiver optical filter is wide enough; and 5) PSK format was used for RF modulation/demodulation, which implies that signal has the same optical power during digital "0" and "1," but with the opposite phase of the RF subcarrier. Because of the page limit, detailed analysis will be reported elsewhere.

A BER of $10^{-9}$ corresponds to $Q=6$. In a system with four subcarriers, the maximum modulation index of each subcarrier is $25 \%$ to avoid clipping. For a $1.75-\mathrm{GHz}$ bandwidth of the receiver low-pass filter (typical for 2.5-Gb/s digital system) and 5-dB erbium-doped fiber amplifiers noise figure, the receiver sensitivity at $10^{-9}$ BER level is approximately $-31 \mathrm{dBm}$. The difference of approximately $4 \mathrm{~dB}$ between the ideal case theoretical prediction and the measured receiver sensitivity, can be mainly attributed to signal waveform distortion and smaller modulation index than $25 \%$ per channel.

In transmission systems with cascaded optical amplifiers, both fiber nonlinearity and accumulated fiber amplifier noise have to be taken into account. Our comprehensive computer simulations reveal that, an SCM system with $2.5 \mathrm{~Gb} / \mathrm{s}$ per SCM channel can tolerate up to $8500 \mathrm{ps} / \mathrm{nm}$ of accumulated dispersion (equivalent to $500 \mathrm{~km}$ of SMF).

In conclusion, we have demonstrated a four-channel digital SCM system with the aggregated capacity of $10 \mathrm{~Gb} / \mathrm{s}$. Optical SSB modulation allows the system to be able to tolerate large amounts of dispersions and increases the efficiency of optical bandwidth utilization. The minimum of $4.7-\mathrm{GHz}$ channel spacing was found to be feasible with commercially available electrical filters.

\section{REFERENCES}

[1] M. R. Phillips and T. E. Darcie, "Lightwave video transmission," in Optical Fiber Telecommunications, I. P. Kaminon and T. L. Koch, Eds. New York: Academic, 1997, vol. IIIA.

[2] P. M. Hill and R. Olshansky, "A 20-channel optical communication using subcarrier multiplexing for the transmission of digital video signals," J. Lightwave Technol., vol. 8, pp. 554-560, Apr. 1990.

[3] K. P. Ho et al., "Hybrid wavelength-division-multiplexing systems for high-capacity digital and analog trunking applications," IEEE Photon. Technol. Lett., vol. 10, pp. 297-299, Feb. 1998.

[4] P. A. Greenhalgh, R. D. Abel, and P. A. Davies, "Optical prefiltering in subcarrier systems.," Electron. Lett., vol. 28, p. 1850, Sept. 1992.

[5] R. Hui, B. Zhu, R. Huang, C. Allen, K. Demarest, and D. Richards, "10 $\mathrm{Gb} / \mathrm{s}$ SCM system using optical single side-band modulation," in Opt. Fiber Commun. Conf. OFC'2001, Anaheim, CA, Paper MM4.

[6] M. E. Frerking, Digital Signal Processing in Communication Systems. London, U.K.: Chapman \& Hall, 1994.

[7] M. Sieben, J. Conradi, D. Dodds, B. Davis, and S. Walklin, "Optical signal sideband (OSSB) transmission for dispersion avoidance and electrical dispersion compensation in microwave subcarrier and baseband digital systems," Electron. Lett., vol. 33, pp. 971-973, May 1997.

[8] G. H. Smith, D. Novak, and Z. Ahmed, "Overcoming chromatic dispersion effects in fiber-wireless systems incorporating external modulators," IEEE Trans. Microwave Theory Tech., vol. 45, pp. 1410-1415, Aug. 1997. 